\title{
Quick starting contraception after emergency contraception: have clinical guidelines made a difference?
}

\author{
Janine Simpson, ${ }^{1}$ Julie Craik, ${ }^{2}$ Louise Melvin ${ }^{3}$
}

\begin{abstract}
'ST1, Community Sexual and Reproductive Health, NHS Greater Glasgow and Clyde, Sandyford Sexual Health Service, Glasgow, UK

${ }^{2}$ Researcher, Faculty of Sexual \& Reproductive Health Clinical Effectiveness Unit, Glasgow, UK ${ }^{3}$ Consultant, Community Sexual and Reproductive Health, NHS Greater Glasgow and Clyde, Sandyford Sexual Health Service, Glasgow, UK, and Clinical Director, Faculty of Sexual \& Reproductive Health Clinical Effectiveness Unit, Glasgow, UK
\end{abstract}

Correspondence Dr Janine Simpson, Sandyford Initiative, 2-6 Sandyford Place, Glasgow G3 7NB, UK;

janine.simpson@hotmail.co.uk

Received 19 April 2013 Revised 6 February 2014 Accepted 26 February 2014 Published Online First 4 April 2014

\section{CrossMark}

To cite: Simpson J, Craik J, Melvin L. J Fam Plann Reprod Health Care 2014;40: 184-189.

\section{ABSTRACT}

Objective When initiating contraception after emergency contraception (EC), conventional practice had been to wait until the next menses. Since 2010, UK guidelines have endorsed quick starting (QS) contraception, namely offering immediate start when requested. We conducted an audit to assess clinical practice before and after QS guidance publication.

Methods A full cycle audit was performed on the clinical notes of women requesting EC during two 2-month periods in 2010 and 2011 in an Integrated Sexual Health Service. All case notes were identified using the National Sexual Health database of sexual health records (Scotland). Information was collated and interpreted using Microsoft Excel and SPSS V.17. Results During January and February 2010 and 2011, 190 and 180 women, respectively, attended for EC, of whom 96 and 97 were identified as potential quick starters. Between 2010 and 2011, a statistically significant increase in QS practice was noted from $20.8 \%(n=20)$ to $37.1 \% \quad(n=36)$ $(p=0.011)$, with a corresponding decrease in the percentage of women traditionally started on hormonal contraception (HC): $24 \%(n=23)$ and $14.6 \%(n=14)$, respectively. There was also a decrease in those advised to return for commencement of HC [55.2\% ( $n=53)$ vs $49 \%$ $(n=47)]$. Of those advised to return, $26.4 \%(n=14)$ and $31.9 \%(n=15)$ had no further contact with the service within at least 6 months.

Conclusions QS practice increased after the introduction of clinical guidelines. However, overall provision of $\mathrm{HC}$ remained low, with only around half of women prescribed a hormonal method.

\section{INTRODUCTION}

When starting non-barrier contraception after emergency contraception (EC), conventional practice had been to advise that women should wait until their next menses. However, since 2010, UK guidance $^{1}$ has advised that immediate 'quick

\section{Key message points}

- Among women prescribed emergency contraception (EC) in an integrated sexual health clinic, few return for ongoing contraception when requested.

- This study suggests a small increase in quick starting (QS) of hormonal contraception since introduction of clinical guidance endorsing QS practice, but little impact on overall uptake of effective contraception.

- When supplying EC, clinicians should discuss ongoing contraceptive needs, offer to supply contraception, and consider QS the woman's preferred contraceptive or an interim 'bridging' method.

- Further research into QS practice is required to understand variations in practice and to confirm potential benefits in terms of reduction in unintended pregnancies.

starting' (QS) of contraception can be offered. This is defined as initiating contraception at the time requested, which may be considered in situations where a woman is likely to continue to be at risk of pregnancy.

Women can also be temporarily bridged with the QS approach until pregnancy can be excluded, and a long-acting method initiated. ${ }^{1}$ Clinical practice has varied among UK contraception providers because QS is outside the terms of contraceptive product licences and had not been formally endorsed until publication of the UK guidance by the Faculty of Sexual \& Reproductive Healthcare (FSRH) in September 2010.

A Cochrane review by Cheng et al. ${ }^{2}$ demonstrated that those women who have 
further sexual intercourse in the same menstrual cycle following EC have a 2-3-fold greater risk of pregnancy than those who abstain. QS has therefore been trialled with adolescents in the USA since 2002 with the aim of improving compliance. ${ }^{3}$ Studies have shown that QS is acceptable to women, ${ }^{4}$ but researchers found limited evidence of reduction of unintended pregnancy rates or an increase in contraception continuation rates.

Following the release of the FSRH guidance a full audit cycle was undertaken to assess QS practice and whether it had changed following guidance publication. Data were collected and collated from two time intervals, before and after guidance publication, at an Integrated Sexual Health Service in Scotland.

\section{METHODS}

Contraceptive prescribing practice following EC administration was examined during two 2-month periods: 1 January-28 February in 2010 and again in 2011.

Following the first period of data collection, an education session detailing current practice and the new FSRH QS guidance was held at a multidisciplinary team meeting.

For each time period total prescriptions of all EC were identified using the National Sexual Health $(\mathrm{NaSH})$ database of sexual health records (Scotland). These records contain a combination of free text notes and EC proforma.

There were 212 (2010) and 222 (2011) EC prescriptions. Where a woman received EC more than once, we analysed only the first prescription and classified the remaining EC prescriptions as additional. The search criterion was the prescription of EC, either levonorgestrel (LNG) or ulipristal acetate (UPA). We excluded those women who had an intrauterine device inserted for EC. There were 190 qualifying case records in 2010 and 180 in 2011, giving a total of 370 records for assessment.

Only LNG prescriptions were used within the first time frame, as UPA prescribing only began in the clinic in June 2010.

Each electronic patient record was individually assessed by the medical researcher and the information collated included:

- Age of woman

- Parity

- Type of EC supplied

- Job title of most senior health practitioner involved in the case

- Whether there was documented discussion of contraception

- Which contraceptive method, if any, was supplied or advised

- Whether advice was given about need for additional contraception

- Whether advice on additional contraception was correct

- Whether condoms were offered or supplied
- Any reasons for not supplying contraception

- When the clients were advised to start the contraceptive method

- Any documented evidence of pregnancy since supply of EC (review of case notes at 6 months post-EC prescription).

Data were entered into the database using Microsoft Excel 2007 and SPSS V.17. SPSS was used to perform descriptive statistics. A $Z$ test was used to compare changes in practice from 2010 to 2011 . Figure 1 demonstrates the study methodology.

The study's main outcome measure was:

- The percentage of women quick started on contraception.

The secondary outcome measures were:

- Those not prescribed hormonal contraception (HC) and advised to return

- Those given HC and advised to start HC with next menses (i.e. traditional starters)

- Reasons for non-administration of HC.

\section{RESULTS}

\section{Demographics}

A total of 190 and 180 women were prescribed EC in 2010 and 2011, respectively. Their mean ages were 22.1 (range 13-45) and 21.7 (14-46) years, respectively. Parity did not affect prescribing practice. In 2010, all women received LNG. In 2011, 89.4\% $(n=161)$ and $10.6 \%(n=19)$ received LNG and UPA, respectively.

\section{HC provision following EC}

Of the 370 women, $86.3 \% \quad(n=164)$ and $84.4 \%$ $(n=152)$ were identified as not already using HC and thus eligible for QS. Of these, $70.1 \%(n=115)$ and $65.1 \%(n=99)$ did not receive any HC with their first EC prescription (Figure 1). Reasons for nonadministration are documented in Table 1.

In 2010, 49 (29.9\%) women were provided with HC. The corresponding figure in 2011 was 53 (34.9\%) women. Data on starting advice were not recorded and therefore excluded for six (12.2\%) and three (5.7\%) women in 2010 and 2011, respectively.

In 2010 and 2011, 23/43 (55.5\%) and 14/50 (28\%) women were started on $\mathrm{HC}$ at the next menses. Quick starters therefore equated to $46.5 \% \quad(n=20)$ and $72.5 \%(n=36)$ of those given HC in 2010 and 2011.

There had been a small decrease of $6.2 \%$ in those potential quick starters being advised to return $(55.2 \%, 2010 ; 49 \%, 2011)$ since publication of the QS guidance. A case note review was performed at 6 months for all women after EC prescription. There were no reported pregnancies within the study.

Of the potential quick starters who were advised to return, $26.4 \%(n=14,2010)$ and $31.9 \% \quad(n=15$, 2011) had no further contact with the service, over at least 6 months following EC. Among the women who did return for contraception for other reasons, $71.8 \%$ 


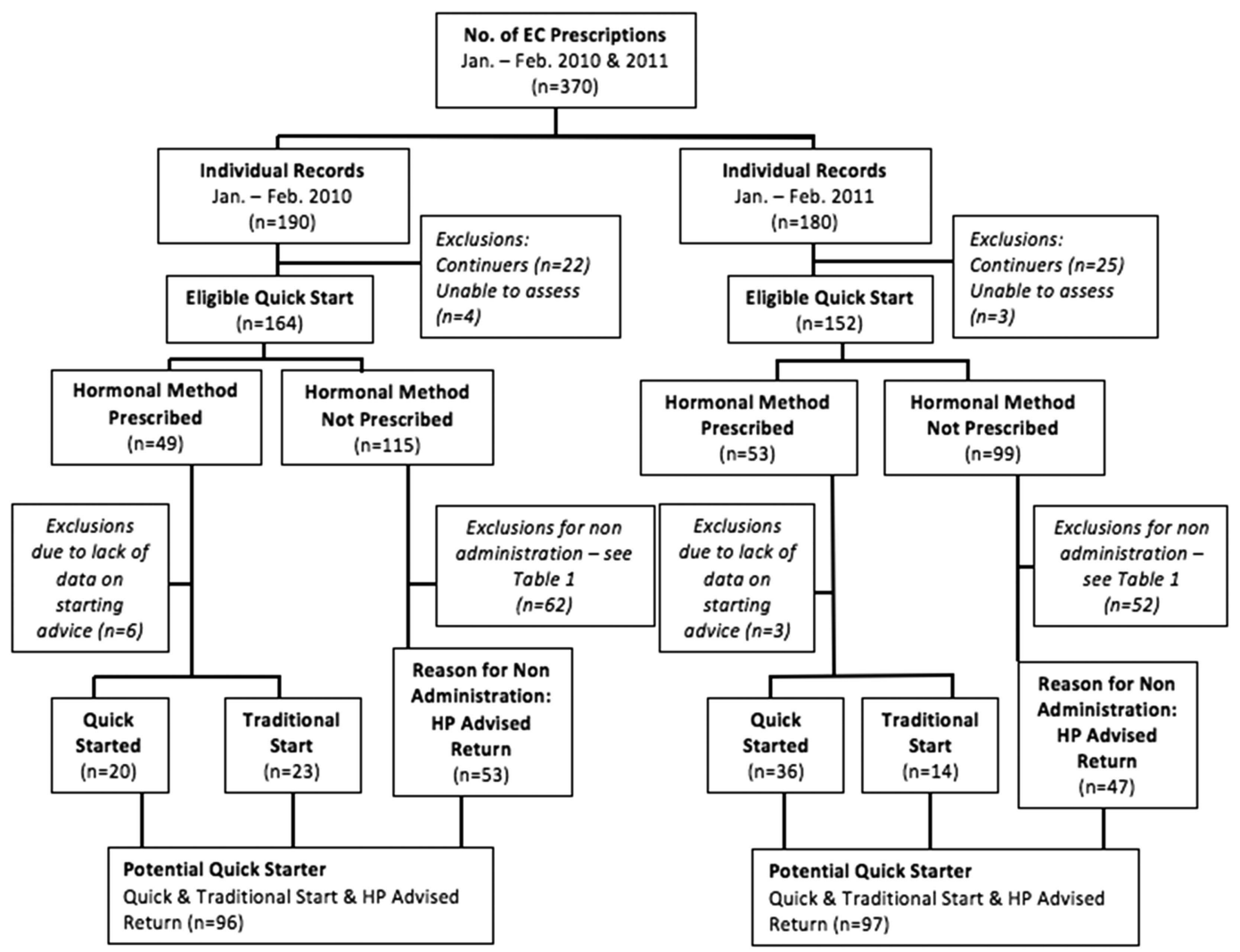

Figure 1 Flowchart detailing audit methodology. Eligible Quick Starter=Not using hormonal contraception, therefore eligible to quick start. Quick Started=Advised to start hormonal contraception (HC) immediately. Traditional Start=Advised to wait until next menses before starting HC. Potential Quick Starter=Quick start+traditional starters+health practitioner advised to return prior to HC administration. Continuer=Already using a method of contraception. EHC, emergency hormonal contraception; HC, hormonal contraception; HP, health practitioner.

$(n=28,2010)$ and $75 \%(n=24,2011)$ did so within 1 month of their EC prescription.

For those advised to return, condoms were offered and accepted by $83.9 \%(n=26)$ and $97.1 \%(n=33)$ of women in 2010 and 2011, respectively.

\section{Contraceptive method supplied}

Table 2 shows the method of contraception supplied on the second visit to those women who returned.

All potential quick starters $(n=96,2010$ and $n=97$, 2011) had the option to be quick started or even bridged onto long-acting reversible contraception (LARC). ${ }^{1}$ In 2010 and 2011, the majority of women

Table 1 Reasons for non-administration of hormonal contraception

\begin{tabular}{|c|c|c|c|c|}
\hline \multirow[b]{2}{*}{ Reason } & \multicolumn{2}{|c|}{2010} & \multicolumn{2}{|c|}{2011} \\
\hline & $n$ & $\%$ & $n$ & $\%$ \\
\hline Not desired by patient & 22 & 19.1 & 32 & 32.3 \\
\hline Patient already has supply & 3 & 2.6 & 11 & 11.1 \\
\hline Health practitioner advised return & 53 & 46.1 & 47 & 47.5 \\
\hline Not recorded & 37 & 32.2 & 9 & 9.1 \\
\hline Total & 115 & 100.0 & 99 & 100.0 \\
\hline
\end{tabular}

who returned chose a method that could have been quick started. The women who returned for intrauterine methods (9.4\%, $n=3,2010$ and $17.8 \%, n=5$, 2011) could have been quick started with a bridging method.

Combined hormonal contraception was prescribed to $87 \%$ and $71.4 \%(n=20$ and $n=10)$ of traditional starters in 2010 and 2011, respectively. Only two women (2010) in the entire study were bridged onto a subsequent method. Table 3 highlights the prescribed QS methods.

Table 2 Method of contraception supplied on second visit, following advice to return by health practitioner

\begin{tabular}{|c|c|c|c|c|}
\hline \multirow[b]{2}{*}{ Method } & \multicolumn{2}{|c|}{2010} & \multicolumn{2}{|c|}{2011} \\
\hline & $n$ & $\%$ & $n$ & $\%$ \\
\hline Emergency contraception & 4 & 12.5 & 0 & 0.0 \\
\hline Combined hormonal contraception & 7 & 21.9 & 5 & 17.9 \\
\hline Progestogen-only pill & 2 & 6.3 & 3 & 10.7 \\
\hline Progestogen-only implant & 12 & 37.5 & 10 & 35.7 \\
\hline Progestogen-only injectable & 4 & 12.5 & 5 & 17.9 \\
\hline Levonorgestrel intrauterine system & 1 & 3.1 & 3 & 10.7 \\
\hline Copper intrauterine device & 2 & 6.3 & 2 & 7.1 \\
\hline Total & 32 & 100.0 & 28 & 100.0 \\
\hline
\end{tabular}


Table 3 Contraceptive methods used for quick starting in 2010 and 2011

\begin{tabular}{|c|c|c|c|c|}
\hline \multirow[b]{2}{*}{ Method } & \multicolumn{2}{|c|}{2010} & \multicolumn{2}{|c|}{2011} \\
\hline & $\bar{n}$ & $\%$ & $\bar{n}$ & $\%$ \\
\hline Combined hormonal contraception & 7 & 35.0 & 15 & 41.7 \\
\hline Progestogen-only pill & 2 & 10.0 & 9 & 25.0 \\
\hline Progestogen-only implant & 7 & 35.0 & 9 & 25.0 \\
\hline Progestogen-only injectable & 3 & 15.0 & 3 & 8.3 \\
\hline Levonorgestrel intrauterine system* & 1 & 5.0 & 0 & 0.0 \\
\hline Total & 20 & 100.0 & 36 & 100.0 \\
\hline
\end{tabular}

*Inappropriate method for quick starting.

\section{Changes in prescribing practice between 2010 and 2011}

Figure 2 demonstrates the changes in prescribing practice among the subgroup of potential quick starters $(n=96,2010 ; n=97,2011)$.

Between 2010 and 2011 there was a 6.2\% decrease in the number of women being advised to return for their HC. Similarly there was a decrease in the percentage of clients who were traditionally started on HC: $24 \%(n=23,2010)$ and $14.6 \%(n=14,2011)$. A statistically significant increase in QS practice was observed from $20 / 96(20.8 \%)$ in 2010 to $36 / 97$ (37.1\%) in $2011(p=0.011,95 \%$ confidence interval $-0.288669,-0.0369328, Z$ score $=-2.54)$.

Medical staff remain the majority of health practitioners who initiate QS with 90\% $(n=18)$ and $63.2 \%$ $(n=22)$ in 2010 and 2011, respectively. However, there has been an increase in nursing staff initiating QS, particularly nurse prescribers $[5 \% \quad(n=1)$ vs $27.8 \%(n=10)$ in 2010 and 2011, respectively]. Table 4 outlines this.

Documented discussion of pregnancy testing was found in $85 \%(n=17)$ and $88.6 \%(n=31)$ of women, with the majority QS $(88.2 \%$ and $93.5 \%$, respectively) being given correct or over-cautious advice. Incorrect advice included testing at 2 weeks or only if menses have not occurred 3 weeks following unprotected sexual intercourse (UPSI). Where advice about additional precautions to QS was documented

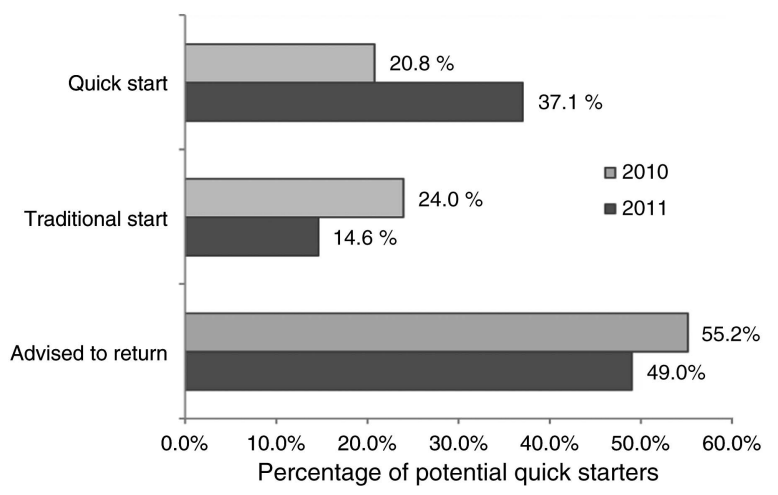

Figure 2 Change in quick starting practice 2010 vs 2011.
Table 4 Quick starting initiation by staff group involvement

\begin{tabular}{lrrrr}
\hline Staff group & \multicolumn{1}{c}{$\boldsymbol{n}$} & \multicolumn{1}{c}{$\boldsymbol{n}$} & \multicolumn{1}{c}{$\%$} \\
\hline Consultant/Associate Specialist/Staff Grade & 13.0 & 65.0 & 20.0 & 55.6 \\
Medical Trainee (SpR SRH, SpR GUM, FY2) & 5.0 & 25.0 & 2.0 & 5.6 \\
Nurse prescriber & 1.0 & 5.0 & 10.0 & 27.8 \\
Nurse PGD & 1.0 & 5.0 & 4.0 & 11.0 \\
Total & 20.0 & 100.0 & 36.0 & 100.0 \\
\hline FY2, Foundation Year Two Doctor; Nurse PGD, Nurse patient group \\
direction; SpR GUM, Specialist Registrar Genitourinary Medicine; SpR SRH, \\
Specialist Registrar Sexual and Reproductive Health.
\end{tabular}

$100 \%(17 / 17)$ and $93.9 \%(31 / 33)$ received the correct information.

\section{DISCUSSION}

There is a paucity of UK data relating to QS HC practice. Clinical practice varies, probably for several reasons such as different attitudes to prescribing outside the product licence; previous lack of clinical guidelines; variable awareness of newly introduced guidelines; and differences in service protocols, patient group directions (PGDs) and availability of support for non-prescribers.

Since the FSRH published its QS guidance we have found a statistically significant increase (16.3\%, $p=0.011)$ in the proportion of eligible women being quick started within this Integrated Sexual Health Service in Scotland.

We have also found a $6.2 \%$ decrease in those women being asked to return for HC, and a $9.4 \%$ decrease in those traditionally started on HC.

Whilst our findings on numbers QS are positive and statistically significant, it is difficult to interpret whether they demonstrate actual clinical improvement in terms of reducing unintended pregnancies.

Our findings are comparable to those of Cameron et al. ${ }^{5}$ who found that $23 \%(n=91)$ of eligible quick starters were initiated on HC following EC $(29.9 \%, n=49$ in 2010 and $34.9 \%, n=53$ in 2011, excluding continuers). Baird ${ }^{6}$ has recently published a study of EC prescribing that includes QS data, but it is difficult to make any direct comparisons because of differences in study design and reporting.

However, even though our QS practice increased, around $50 \%$ of women eligible to be quick started were still advised to return. Although this is not incorrect practice and some clients may have attended their general practioners for contraception or been using condoms, for others this finding might indicate that there was a delay in accessing potentially more reliable methods. Within the free text clinical notes there was no documented evidence as to why women were asked to return rather than being quick started.

Worryingly, approximately one-third of the women who were advised to return had not done so after at least 6 months. This highlights the fact that plans can 
fail when relying on re-attendance, and should encourage health practitioners to be proactive in their consultations. Motivational interview techniques may play a role here.

QS guidance encourages bridging women safely onto their subsequent method of contraception, particularity LARC, rather than prescribing HC for traditional starting. As mentioned earlier, women who have further sexual intercourse in the menstrual cycle following EC are at a 2-3-fold greater risk of pregnancy than those who abstain. ${ }^{2}$ Initiation of LARC is therefore a useful discussion to have with those presenting for EC, as these methods are more effective in reducing unintended pregnancy with typical use. A reluctance to quick start progestogen-only implants was noted in both time frames $(7 / 20$ and 9/36 in 2010 and 2011, respectively). This method is longeracting than oral methods, and it may be that although its use is supported by the FSRH, ${ }^{1}$ practitioners are more reluctant to initiate a method that is more invasive. This method also requires returning for removal should a pregnancy be identified that the woman wishes to continue. Cost issues may also play a role.

Amongst those women who were prescribed $\mathrm{HC}$ at the second visit, the majority were given a QS method in accordance with FSRH guidance. ${ }^{1}$ Surprisingly, only two women in the entire audit were bridged onto their subsequent method of LARC.

Two women within the audit study were given incorrect advice regarding pregnancy testing: test after 2 weeks or only if no menses 3 weeks post-UPSI. Pregnancy testing should always be done 3 weeks following UPSI, irrespective of bleeding pattern, as unscheduled bleeding is common and can be misleading. Furthermore, two women in 2011 were given incorrect advice regarding additional precautions, with fewer than 7 days advised, when 7 days were required. This could have led to unintended pregnancy and would have defeated the purpose of QS. However, within the study there were no reported pregnancies, although it is possible that women may have presented elsewhere. Therefore it is fundamental that the health practitioner provides correct advice, particularly when prescribing outside the product licence.

Within the service audited, medical staff, nurse prescribers and nurses via PGD may prescribe EC and HC. $^{7-9}$ PGD limits staff to strict criteria. At the time of the audit the PGD did not allow QS contraception following EC administration. During both phases of the audit a doctor was available as a central support for all staff, particularly at the peripheral nurse-led clinics. Our service allows electronic prescribing; therefore nursing staff bound by a PGD should be empowered to discuss these cases with staff who can quick start. This audit suggests that QS could perhaps be facilitated by inclusion of QS in PGDs and/or better utilisation of the supporting doctor.
Given the nature of the specialised service it is difficult to know if these results are generalisable across other services such as general practice. In addition, local clinical practice may have been influenced by the presence of the guidance authors (the FSRH Clinical Effectiveness Unit) within the same clinical service and may therefore not be representative of other sexual health clinics.

Other limitations to the study include the retrospective nature of the audit, for example, possible missing data, and its reliance on descriptive data, which may not reflect the consultation.

Case notes were reviewed by different primary medical researchers in 2010 and 2011, which may have led to slight interpretational differences. All data were, however, checked for consistency prior to analysis. The information gained from $\mathrm{NaSH}$ was from a combination of free text and the pre-set EC proforma, both of which have varying usage. Within the EC proforma there is no mention of ongoing contraception. This may also be a missed opportunity to remind staff to consider QS.

\section{CONCLUSIONS}

QS practice was shown to increase in an integrated sexual health setting following publication of national guidelines. It is uncertain whether practice changed as a result of the FSRH guidance and whether the change is clinically significant in terms of reducing unintended pregnancy. It is obvious that health practitioner training and education are important when considering how to improve QS practice, along with motivating women to consider HC or bridging onto their chosen method. Clearly we cannot rely on all women returning for HC and we should therefore be proactive in all consultations. Further auditing of practice following EC administration may also help our understanding, allow services to implement changes and monitor whether improvement in practice is sustained.

Acknowledgements The authors gratefully acknowledge the assistance of Dr Susanna Hall, Dr Tim Wright, Mr Martin Stevenson and Dr David Young with data collection and analysis.

Competing interests Julie Craik and Louise Melvin work in the FSRH Clinical Effectiveness Unit and were members of the guideline development group for the FSRH guidance on quick starting hormonal contraception.

Ethics approval NHS Greater Glasgow and Clyde Caldicott Guardian Approval was sought and granted on 12 October 2012

Provenance and peer review Not commissioned; externally peer reviewed.

\section{REFERENCES}

1 Faculty of Sexual \& Reproductive Healthcare Clinical Effectiveness Unit. Quick Starting Contraception. 2010. http:// www.fsrh.org/pdfs/CEUGuidanceQuickStartingContraception. pdf [accessed 4 March 2012].

2 Cheng L, Gulmezoglu AM, Van Look PFA. Interventions for emergency contraception. Cochrane Database Syst Rev 2008;2: CD001324. 
3 Lara-Torre E. "Quick Start", an innovative approach to the combination oral contraceptive pill in adolescents. Is it time to make the switch? J Pediatr Adolesc Gynecol 2004;17:65-67.

4 Lopez LM, Newmann SJ, Grimes DA, et al. Immediate start of hormonal contraceptives for contraception. Cochrane Database Syst Rev 2008;2. doi: 10.1002/14651858. CD006260.pub2 [accessed 4 March 2012].

5 Cameron ST, Glasier A, Johnstone A, et al. Ongoing contraception after use of emergency contraception from a specialist contraceptive service. Contraception 2011;84:368-371 [accessed 4 March 2012].

6 Baird AS. Use of ulipristal acetate, levonorgestrel and the copper intrauterine device for emergency contraception following the introduction of new FSRH guidelines. J Fam Plann Reprod Health Care 2013;39:264-269.

7 NHS Greater Glasgow and Clyde. Sandyford Services. Clinical Management and Protocols. http://www.sandyford.org/ clinicians/clinical-management-and-protocols.aspx [accessed 4 March 2012].

8 NHS Greater Glasgow and Clyde. Patient Group Direction (PGD) for Nurses and Midwives. Combined Hormonal Contraception. October 2011. GG\&C PGD ref no: 2011/909 [accessed 4 March 2012].

9 NHS Greater Glasgow and Clyde. Patient Group Direction (PGD) for Nurses and Midwives. Levonorgestrel. April 2011. GG\&C PGD ref no: 2011/851 [accessed 4 March 2012]. 